3 CHUNG, J.E, JENG, M.C., MOON, J.E., KO, P.K., and HU, C. 'Performance and reliability design issues for deep-submicrometer

4 The simulations were performed using aTLAS/BLAZE, SILvaCO International, Version 1.0, Copyright 1993

\section{Saturation region model for a-Si:H TFTs using a quasi-two-dimensional approach}

J.B. Kuo and S.S. Chen

Indexing terms: Semiconductor devices and materials

A saturation region model for a-Si:H thin film transistors using quasi-two-dimensional approach is reported. As verified by the published data, this analytical saturation region model provides an accurate prediction of the the drain current characteristics of an a-Si:H TFT.

Introduction: Recently, a-Si:H TFTs have been receiving much attention owing to their on-chip integration capabilities with complex arrays of a-Si:H LCDs and other image-sensing arrays [1]. To facilitate circuit design for a-Si:H digital control circuits, an effective saturation region model for a-Si:H TFTs is very important Compared to an MOS device, the internal condition of an a-Si:H TFT may be quite different due to the existence of the trapped charges in localised deep and tail states in the amorphous silicon. Shur and Hack [2] reported an analytical saturation region model based on an 'nin diode' approach, where the region after pinchoff is regarded as an intrinsic region. Recently, using an effective tem perature approach, an analytical triode-region model has been derived [3]. In this Letter, by applying the quasi-two-dimensional approach $[4,5]$, an analytical saturation region model for the aSi:H TFT is described. In the following Section, derivation of the analytical model is described first, followed by comparison with experimental data and discussion.

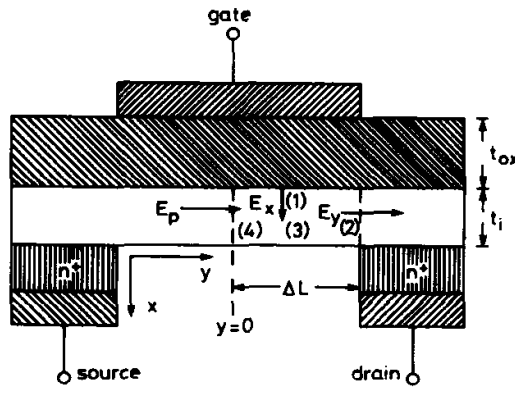

G4in

Fig. 1 Cross-section of a-Si:H under study

Derivation of saturation region model: Consider an a-Si:H TFT with its cross-section near the drain area with its co-ordinates as shown in Fig. 1. Following the quasi-two-dimensional approach, Fig. 1, we obtain

$$
\oint_{s} \vec{D} d \vec{l}=-q \int_{0}^{y} \int_{0}^{t_{i}}\left(n_{l o c}+n_{f+e e}\right) d x d y
$$

where $\overrightarrow{\mathbf{D}}$ is the electric displacement density perpendicular to the path. $t_{i}$ is the thickness of the amorphous thin film. Considering the path of line integration, we obtain

$$
\begin{gathered}
\int_{0}^{t_{i}}\left(\frac{d \Psi_{s}}{d y}+E_{p}\right) d x+\int_{0}^{y} \frac{C_{o x}}{\epsilon_{s}}\left(V_{g}-V_{f b}-\Psi_{s}\right) d y \\
=\frac{q}{\epsilon_{s}} \int_{0}^{y}\left(N_{l o c}+N_{f r e e}\right) d y
\end{gathered}
$$
MOSFET's', IEEE Trans. March 1991, ED-38, (3) pp. 545-554 applying the Gauss law in the region near the drain as shown in

$$
\frac{d^{2} \Psi_{s}}{d y^{2}}=\frac{C_{o x}}{\epsilon_{s} t_{i}}\left(\Psi_{s}-\left(V_{g}-V_{f b}\right)\right)+\frac{q}{\epsilon_{s} t_{i}}\left(N_{l o c}+N_{\text {free }}\right)
$$

Using the formula

$$
2 \frac{d \Psi_{s}}{d y}\left(\frac{d^{2} \Psi_{s}}{d y^{2}}\right)=\frac{d}{d y}\left(\frac{d \Psi_{s}}{d y}\right)^{2}
$$

from eqn. 3, we obtain

$$
\begin{aligned}
E_{y d}^{2} & =E_{p}^{2} \\
& +\frac{C_{o x}}{\epsilon_{s} t_{i}}\left(\left(\Psi_{s d}-\left(V_{g}-V_{f b}\right)\right)^{2}-\left(\Psi_{s p}-\left(V_{g}-V_{f b}\right)\right)^{2}\right) \\
& +\frac{2 q}{\epsilon_{s} t_{i}} \int_{\Psi_{s p}}^{\Psi_{s d}}\left(N_{l o c}+N_{\text {free }}\right) d \Psi_{s}
\end{aligned}
$$

where $\Psi_{s d}$ is the potential at the drain end, $\Psi_{s p}$ is the potential at the pinchoff point. $\mathrm{E}_{p}$ is the $y$ direction lateral electric field at the pinchoff point.

The total charges in localised states and the total free carriers are related by the following equation $[6,7]: N_{\text {free }} /\left(N_{\text {froe }}+N_{b x}\right)=$ $\mu_{v} / \mu_{\text {fet }}$ where $\mu_{\text {fet }}$ is the field effect mobility, and $\mu_{v}$ is the band mobility. $\mu_{d} / \mu_{\text {fet }}$ is a function of $V_{g}$. From eqn. 4 ,

$$
\begin{gathered}
E_{y d}^{2} \cong E_{p}^{2}-\frac{C_{o x}}{\epsilon_{s} t_{i}} \Psi_{s o}^{2}+\frac{C_{o x}}{\epsilon_{s} t_{i}}\left(\Psi_{s d}-\left(V_{g}-V_{f b}\right)\right)^{2} \\
+\frac{2}{\epsilon_{s} t_{i}} \frac{1}{\mu_{f e t}} \frac{L_{e f f}}{W}\left(I_{d}-I_{d s s}\right)
\end{gathered}
$$

where $L_{e f}$ is the effective channel length. The drain current $I_{d}$ and the drain current when pinchoff at drain starts to occur $I_{d s r}$ are defined as

$$
\begin{aligned}
I_{d} & =\frac{W}{L_{e f f}} \mu_{o} \int_{0}^{\Psi_{s d}} q N_{f r e e}\left(\Psi_{s}\right) d \Psi_{s} \\
I_{d s s} & =\frac{W}{L_{e f f}} \mu_{o} \int_{0}^{\Psi_{s p}} q N_{f r e e}\left(\Psi_{s}\right) d \Psi_{s}
\end{aligned}
$$

The output conductance, which is defined as the derivative of the drain current with respect to the drain potential, becomes

$$
g_{0}=\frac{I_{d s s} L_{e f f}}{\left(L_{e f f}-\Delta L\right)^{2}} \frac{\partial \Delta L}{\partial V_{d}}
$$

where

$$
\Delta L=\int_{\Psi_{s p}}^{\Psi_{s d}} \frac{d \Psi_{s d}}{E_{y d}}
$$

Because the a-Si:H TFT under study is a long channel device, in the region after pinchoff, the electrons are not travelling at saturated velocity. As a result, the lateral electric field at the pinchoff point is

$$
E_{p}=\frac{I_{d}}{q \mu_{o} W N_{\text {free }}\left(\Psi_{s o}\right)}
$$

as described in a previous paper [3].

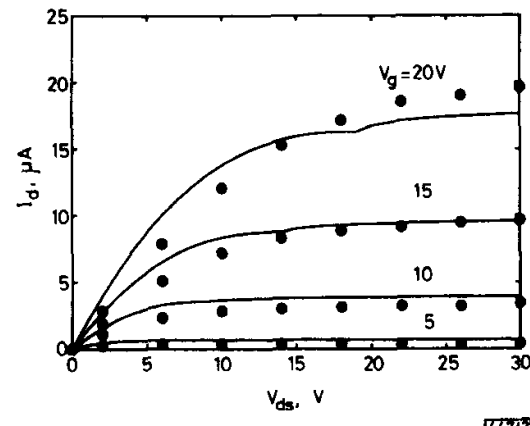

Fig. 2 Drain current characteristics of the a-Si:H TFT under study based on the analytical model and the published data

\section{our model}

measured

From eqn. 2, we obtain

ELECTRONICS LETTERS 14th October 1993 Vol. 29 No. 21 


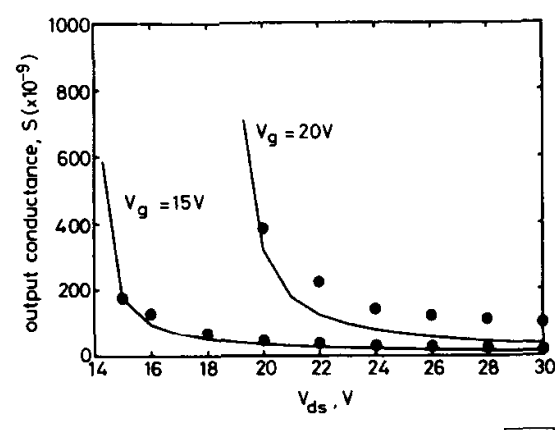

区47/3

Fig. 3 Output conductance against $V_{d s}$ of a-Si:H TFT biased at $V_{g}=$

\section{our model}

Comparison with experimental data, and discussion: To verify the analytical drain model using the effective temperature approach, an a-Si:H TFT with a channel length of $8 \mu \mathrm{m}$ and a channel width $80 \mu \mathrm{m}$ has been used in the study. The a-Si:H TFT has an a-Si thin film of $500 \AA$ and a gate oxide of $3000 \AA$. The band mobility $\mu_{0}$ is $17 \mathrm{~cm}^{2} /$ Vs. The effective density of states $N_{c}$ is $7 \times 10^{19} \mathrm{~cm}^{-3}$. Fig. 2 shows the drain current characteristics of the a-Si:H TFT based on the analytical model using the quasi-two-dimensional approach and the effective temperature approach and the published data [2] $\left(L_{e y j}=7.2 \mu \mathrm{m}\right)$. A close fit between the model results and the published data can be observed. Fig. 3 shows the output conductance against $V_{d}$ of the a-Si:H TFT biased at $V_{g}=15 \mathrm{~V}$ $20 \mathrm{~V}$ based on the analytical model and the measured data. A shown in this Figure, compared to the single-crystalline MOS device, the output conductance is two orders of magnitude smaller due to much smaller mobility.

() IEE 1993

Electronics Letters Online No: 19931229

13 September 1993

J. B. Kuo and S. S. Chen (Dept. of Electrical Eng., National Taiwo University, Roosevelt Rd., \#I, Sec. 4, Taipei, Taiwan 106-17, Republic of China)

\section{References}

1 thompson, M.J., and TUAN, H.C: IEDM Tech. Dig., 1986, (Los Angeles), p. 192

2 SHUR, M.S., HACK, M, and SHAW, J.G.: "A new analytic model for amorphous silicon thin-film transistors', $J$. Appl. Phys., October $1989,66,(7)$, pp. 3371-3380

3 KUO, J.B., and CHEN, C.S.: "Analytical drain current model for aSiH:H TFTs by simultaneously considering localised deep and tail states', Electron. Lett., 1993, 29, (17)

4 KO, P.K., MULLER, R.S., and HU, C.: 'A unified model for hot-electron currents in MOSFET's'. IEDM Tech. Dig., December 1981, pp 600-603

5 CHOW, H.C., FENG, w.S., and KUO, J.B.: 'An improved analytical short-channel MOSFET model valid in all regions of operation for aanalog/digital circuit simulation', IEEE Trans. Computer Aided Design, December 1992

6 SHUR, M., and HACK, M.: 'Physics of amorphous silicon based alloy field-effect transistors', J. Appl. Phys., May 1984, 55, (11), pp 3831-3842

7 SHUR, M., and HACK, M.: 'Capacitance-voltage characteristics of amorphous silicon thin-film transistors', J. Appl. Phys., 1989, 66, pp. 338-3385

\section{Genetic design of minimum-time controllers}

B. Porter and S.S. Mohamed

\section{Indexing terms: Genetic algorithms. Controllers}

In practice, minimum-time control problems are notoriously difficult to solve. It is therefore shown that a two-level hierarchy

of genetic algorithms can be readily used to solve such problems.

Introduction: The design of minimum-time controllers is an important aspect of many tasks in industrial automation. Thus, for example, driving industrial robots as rapidly as possible is an important means of increasing industrial productivity. It is for this reason that the design of minimum-time controllers continues to be an important issue in control engineering 40 years after the initial pioneering work in this field described by Athans and Falb [1] , for example. However, notwithstanding the theoretical possibility of solving minimum-time control problems by using such methodologies as the celebrated minimum principle of Pontryagin [2] , the practical difficulties of solving such problems are still immense.

However, in this Letter it is indicated that genetic algorithms $[3,4]$ provide an alternative approach to the design of minimumtime controllers that is simple, direct and readily able to take into account hard constraints on controller outputs. It is shown that two genetic algorithms (operating locally and globally, in tandem) are required. These fundamental results are described by reference to a simple system, in order to make the exposition as clear as possible. But it is important to note that the same basic approach is equally applicable to the solution of complex problems in the minimum-time control of robots [5] and of spacecraft [6].

Genetic design methodology: In order to describe clearly the genetic design methodology for minimum-time controllers, it is convenient to consider the first-order, single-input/single-output plant governed by the state equation

$$
\dot{x}(t)=u(t)
$$

where the dot denotes differentiation with respect to $t$. It is desired to find the control strategy, sublect to the hard constraint $|u(t)| \leq$ $\alpha$, which drives this plant from any initial state $x(0)=x_{0}$ to the origin $x\left(t_{f}\right)=0$ in minimum time. The genetic solution of this minimum-time control problem is greatly facilitated by the introduc tion of a dimensionless time variable $\tau=t / t$, where $t_{f}$ is the final time whose minimum value is to be determined. It is then evident that eqn. 1 assumes the form

$$
x^{\prime}(t)=t_{f} u(\tau)
$$

where the prime denotes differentiation with respect to $\tau$.

In terms of eqn. 2 , the minimum-time control problem can be solved as follows:

(i) Select $t_{f}$

(ii) Determine, for each selected value of $t$, the control strategy $u(\tau)(0 \leq \tau \leq 1),|u(\tau)| \leq \alpha$, such that the final positional error $|x(1)|$ is minimal.

(iii) Repeat this process with different values of $t_{f}$ until the minimal $t_{f}$ such that $|x(1)|=0$ is obtained.

The resulting value of $t_{f}$ is the required minimum final time, and the associated control, $u(t)(0 \leq t \leq t),|u(t)| \leq \alpha$, is the required minimum-time control strategy.

This formulation indicates that a two-level hierarchy of genetic algorithms can be used to design minimum-time controllers in the following way:

(i) Use a local genetic algorithm (GA), to determine the control strategy $u(\tau)(0 \leq \tau \leq 1),|u(t)| \leq \alpha$, that minimises the final positional error $|x(1)|$ for each value of $t_{f}$ from a population of $N^{(s)}$ values of $t_{f}$. This local genetic algorithm uses a fitness function

$$
\phi_{\ell}=1 /|x(1)|
$$

a population of $N^{\text {.) }}$ values of $u$ for each value of $t_{f}$ and evolves 